\title{
Herpes Zoster Ophthalmicus Presenting as Acute Orbital Inflammation Preceding the Vesicular Rash
}

\author{
Dae Woong Bae, MD (D; Jae Young An, MD, PhD (iD \\ Department of Neurology, St. Vincent's Hospital, College of Medicine, The Catholic University of Korea, Seoul, Korea
}

Herpes zoster ophthalmicus (HZO) can affect various structures in the orbit. Here, we report a very rare case of HZO in a 54-year-old male presented with acute orbital inflammation preceding vesicular eruption. Orbital magnetic resonance imaging revealed findings of orbital myositis, dacryoadenitis, and orbital cellulitis in addition to the enhancement of the right trigeminal ophthalmic branch and ultrasonography demonstrated swelling of the right supraorbital nerve. The patient experienced a significant improvement without any residual complications with intravenous acyclovir, oral steroids and ultrasonography-guided supraorbital nerve block. This case demonstrates that HZO can initially present with a wide variety of symptoms, including acute orbital inflammation without a vesicular rash, and the varicella-zoster virus should be considered as a cause of acute orbital inflammation.

\section{J Neurosonol Neuroimag 2020;12(2):91-94}

Key Words: Herpes zoster ophthalmicus; Orbital myositis; Dacryoadenitis; Orbital cellulitis
Received: August 19, 2020

Revised: August 26, 2020

Accepted: October 14, 2020

Correspondence:

Jae Young An, MD, PhD

Department of Neurology, St. Vincent's Hospital, The Catholic University of Korea, 93 Jungbu-daero, Paldal-gu, Suwon 16247 , Korea Tel: $+82-31-881-8876$ Fax: +82-31-243-0306 E-mail: nrjyan@gmail.com
Herpes zoster ophthalmicus (HZO) occurs from reactivation of latent varicella zoster virus in the trigeminal ganglia and represents approximately $10 \%$ to $25 \%$ of all cases of herpes zoster. ${ }^{1}$ Of these cases, $20 \%$ to $70 \%$ have ocular and periocular involvement which includes keratitis, conjunctivitis, upper eyelid edema, blepharoptosis, anterior uveitis, scleritis, and episcleritis. ${ }^{2}$ But acute orbital inflammation, which can manifest as optic neuritis, cranial nerve palsies, and orbital myositis, is less common. It is exceedingly rare for HZO to presented as acute orbital inflammation without a preceding vesicular rash. ${ }^{1-10}$ Herein we report a rare case of $\mathrm{HZO}$ with characteristic magnetic resonance imaging (MRI) and ultrasonographic findings that initially presented with various symptoms of acute orbital inflammation before eruption of a vesicular rash.

\section{CASE}

A 54-year-old man with well-controlled diabetes mellitus visited an ophthalmology clinic with right forehead pain, exophthalmos, periorbital swelling, and conjunctival congestion on the right eye. The ophthalmologist prescribed oral antibiotics with diagnosis of orbital cellulitis. Two days later, the patient had binocular diplopia and right eyeball pain during ocular movement and was referred to our neurology clinic. On physical examination, visual acuity, visual field, pupillary evaluation, and fundoscopy were normal. Extraocular motility of the right eye revealed limitation of vertical and horizontal eyeball movement. There was paresthesia in the area of the right trigeminal ophthalmic nerve. Orbital MRI revealed enhancement of the ophthalmic branch of the right trigeminal nerve, swelling of the soft tissue in the right forehead and periorbital tissue, and enlargement of the lacrimal gland and extraocular muscles (Fig. 1A-C). During di- 
agnostic work-up, 7 days after the onset of symptoms, blistering skin lesions occurred in the right trigeminal ophthalmic branch region. The laboratory results showed serum varicella zoster virus immunoglobulin G (enzyme-linked immunosorbent assay, ELISA) was 3,457 $\mathrm{mIU} / \mathrm{mL}$ (positive $\geq 165.0$ ), and varicella zoster virus immunoglobulin $\mathrm{M}$ (ELISA) was the 1.56 index (positive $\geq 1.10$ ). human immunodeficiency virus $\mathrm{Ag} / \mathrm{Ab}$ were all negative, and $\mathrm{HbAlC}$ was $5.6 \%$. The cerebrospinal fluid analysis revealed a slightly elevated protein level $(61 \mathrm{mg} / \mathrm{dL})$ without pleocytosis. Autoimmune antibody tests for angiotensin-converting enzyme, anti-nuclear antibody, double-stranded DNA, anti-Ro, anti-La, and anti-neutrophil cytoplasmic antibody were all negative. Intravenous acyclovir $30 \mathrm{mg} / \mathrm{kg}$ and oral prednisolone $80 \mathrm{mg}$ were started immediately after the appear- ance of skin lesions. For a few days after the treatment started, the patient complained of severe neuralgic pain around the right forehead, despite treatment with opioids, gabapentin, and nonsteroidal anti-inflammatory drugs. Ultrasonography, performed 5 days after rash onset, revealed swelling of the right supraorbital nerve and ultrasonography-guided supraorbital nerve block was performed (Fig. 1D). After 2 weeks of treatment, the patient experienced a significant improvement without any residual complications. He had no postherpetic neuralgia during the follow-up period of 3 months.

\section{DISCUSSION}

In less common cases, HZO causes a variety of rare
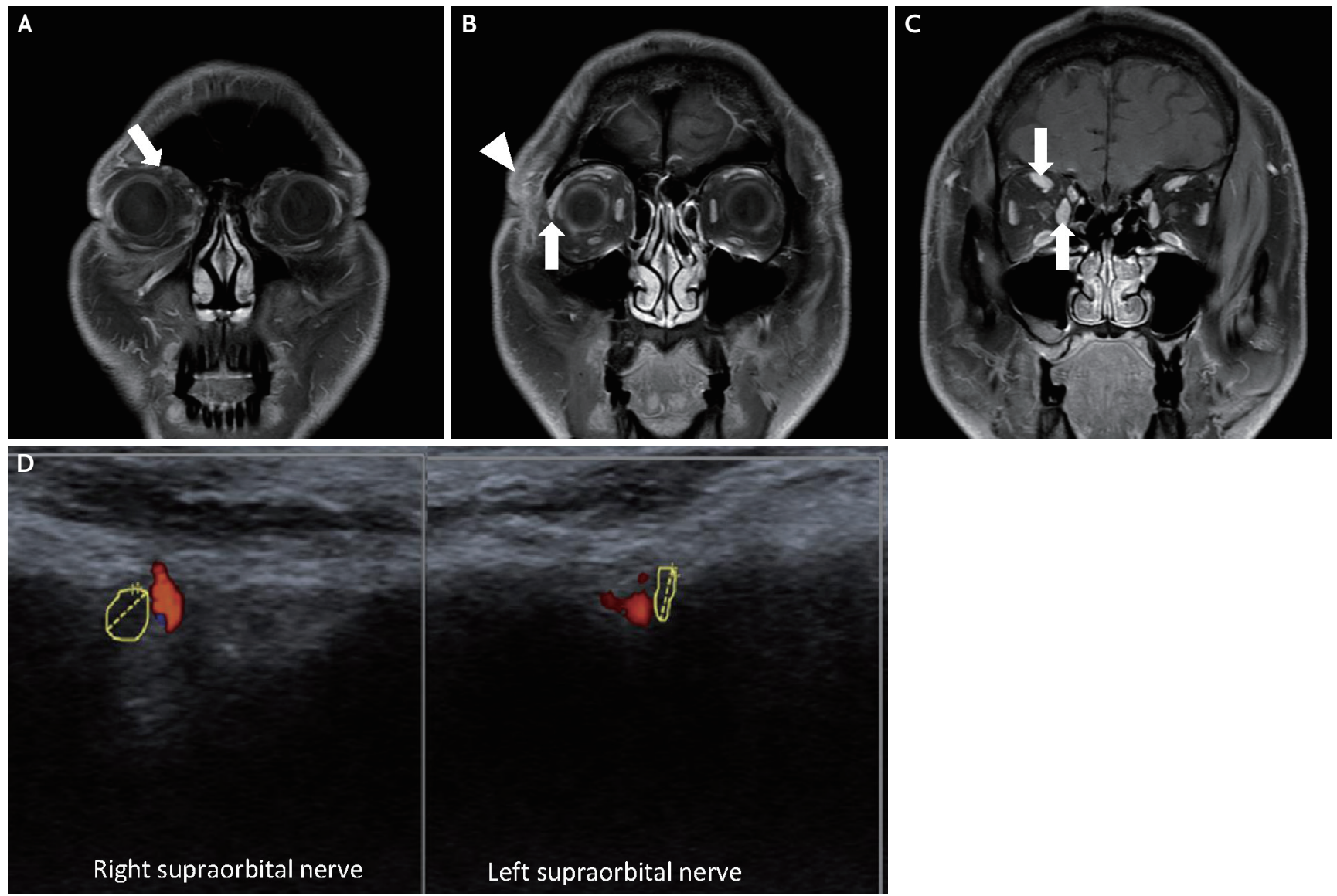

FIG. 1. (A) Coronal and axial images of $T_{1}$ fat suppression contrast magnetic resonance imaging of the orbit showed enhancement of the frontal branch of the right trigeminal nerve (arrow), (B) soft tissue swelling and infiltration in the right forehead and periorbital tissue (arrowhead), enlargement of the lacrimal gland (arrow) and (C) swelling of the right superior rectus and medial rectus muscles (arrows). (D) Ultrasonography revealed swelling of the right supraorbital nerve (cross-sectional area, $3 \mathrm{~mm}^{2}$ ) adjacent to the supraorbital artery in the supraorbital notch compared to the let supraorbital nerve (cross-sectional area, $1 \mathrm{~mm}^{2}$ ). 
ophthalmological and neurological complications. Generally, it is not difficult to make a diagnosis of HZO because a vesicular rash over the forehead precedes other manifestations of HZO. In one study, ocular involvement in $\mathrm{HZO}$ occurred 1 to 4 weeks following the skin eruption. ${ }^{11}$ Rarely, HZO causes various ophthalmological and neurological complications prior to skin lesions. The occurrence of acute orbital inflammation preceding skin rashes was first published in 1991 by Volpe and Shore. ${ }^{8}$ They demonstrated that enlargement of the extraocular muscles on computed tomography scan and symptom onset of acute orbital myositis developed 3 days before skin lesions. To our knowledge, there have been 12 cases of $\mathrm{HZO}$ preceding a skin lesion, ${ }^{1-10}$ and the accompanying reports showed that the interval of zoster skin development after ocular involvement was a mean of 5.8 days (range, 2 to 10 days). In 12 cases, 10 cases showed myositis on the imaging studies, and one case showed ophthalmoplegia without findings of myositis. The other case showed acute dacryoadenitis without ophthalmoplegia or myositis. ${ }^{6}$ In addition to inflammation of the ocular motor cranial nerves, ocular myositis and mechanical limitation of the orbit caused by orbital soft tissue edema have been suggested as important mechanisms for ophthalmoplegia in HZO, but conclusive clinicopathologic correlations are lacking. ${ }^{4}$ Acute dacryoadenitis in HZO is very rare, especially when it precedes the skin lesions. However, previous reports and our case showed enlargement of the lacrimal gland with other symptoms of acute orbital inflammation preceding a skin lesion., ${ }^{7,9,10}$ Acute dacryoadenitis may be obscured by other symptoms, or it can be overlooked because of lack of routine imaging studies in patients with HZO.

The empiric treatment of acute orbital inflammation in HZO is systemic acyclovir and steroids. In the previous reports about $\mathrm{HZO}$ without preceding rashes, six patients of nine cases who received both acyclovir and systemic steroids had a complete resolution of symptoms. ${ }^{3-5,7,8,10}$ Among five patients who received only acyclovir, two patients showed complete resolution. ${ }^{2,6,9}$ Although the effect of systemic steroids on HZO-associated acute orbital inflammation has not been studied with a randomized controlled trial due to the rarity of cases, the combined treatment of acyclovir and systemic steroids usually carries a good prognosis, especially in patients with normal immunity. ${ }^{3}$

This case showed that HZO can present orbital myositis, dacryoadenitis, orbital cellulitis and trigeminal neuritis simultaneously without a vesicular skin lesion. Physicians should consider HZO in patients with acute orbital inflammation that occur within a several days, even though without skin lesions, and appropriate treatment with intravenous acyclovir and systemic steroids with ultrasonography-guided nerve block can have a good prognosis.

\section{Conflicts of Interest}

No potential conflicts of interest relevant to this article was reported.

\section{REFERENCES}

1. Delengocky T, Bui CM. Complete ophthalmoplegia with pupillary involvement as an initial clinical presentation of herpes zoster ophthalmicus. J Am Osteopath Assoc. 2008;108:615-621.

2. Tseng YH. Acute orbital myositis heralding herpes zoster ophthalmicus: report of a case. Acta Neurol Taiwan. 2008;17:47-49.

3. Arda H, Mirza E, Gumus K, Oner A, Karakucuk S, Sirakaya E. Orbital apex syndrome in herpes zoster ophthalmicus. Case Rep Ophthalmol Med. 2012;2012:854503.

4. Kawasaki A, Borruat FX. An unusual presentation of herpes zoster ophthalmicus: orbital myositis preceding vesicular eruption. Am J Ophthalmol. 2003;136:574-575.

5. Kim HT, Moon SY, Lee KH. Acute orbital myositis before Herpes zoster ophthalmicus. Korean J Anesthesiol. 2012;62:295-296.

6. Obata H, Yamagami S, Saito S, Sakai O, Tsuru T. A case of acute dacryoadenitis associated with herpes zoster ophthalmicus. Jpn J Ophthalmol. 2003;47:107-109.

7. Patheja RS, Weaver T, Morris S. Unique case of orbital myositis and dacryoadenitis preceding the vesicular rash of herpes zoster ophthalmicus. Clin Exp Ophthalmol. 2016;44:138-140.

8. Volpe NJ, Shore JW. Orbital myositis associated with herpes zoster. Arch Ophthalmol. 1991;109:471-472.

9. Chiang E, Bajric J, Harris GJ. Herpes zoster ophthalmicus with orbital findings preceding skin rash. Ophthalmic Plast Reconstr Surg. 2018;34:e113-e115. 
10. Bak E, Kim N, Khwarg SI, Choung HK. Case series: herpes zoster ophthalmicus with acute orbital inflammation. Optom Vis Sci. 2018;95:405-410.
11. Harding SP, Lipton JR, Wells JC. Natural history of herpes zoster ophthalmicus: predictors of postherpetic neuralgia and ocular involvement. Br J Ophthalmol. 1987;71:353-358. 